\title{
Implementation of the Chronic Care Model to Reduce Disparities in Hypertension Control: Benefits Take Time
}

\author{
Barbara J. Turner, MD, MSED ${ }^{1,2}$, Julie A. Parish-Johnson, MS, LCDC, CRC², Yuanyuan Liang, PhD 2,3, \\ Tracy Jeffers, $B S^{4}$, Shruthi V. Arismendez, $M S^{2}$, and Ramin Poursani, $\mathrm{MD}^{5}$
}

\begin{abstract}
'Department of Medicine, Joe R. and Teresa Lozano Long School of Medicine, University of Texas Health San Antonio (UT Health San Antonio), San Antonio, TX, USA; ${ }^{2}$ Center for Research to Advance Community Health (ReACH), UT Health San Antonio, San Antonio, TX, USA; ${ }^{3}$ Department of Epidemiology and Public Health, University of Maryland School of Medicine, Baltimore, MD, USA; ${ }^{4}$ Information and Technology Services, University Health System, San Antonio, TX, USA; ${ }^{5}$ Department of Family and Community Medicine, Joe R. and Teresa Lozano Long School of Medicine, UT Health San Antonio, San Antonio, TX, USA.
\end{abstract}

BACKGROUND: The Chronic Care Model (CCM) has been endorsed by experts to reduce disparities in chronic disease outcomes but benefits may be slow to appear in lowincome populations.

OBJECTIVE: To evaluate the effect of CCM implementation on systolic blood pressure (SBP) control in minority patients with diabetes mellitus (DM).

DESIGN: Retrospective study from 2012 to 2016 in two primary care clinics with primarily uninsured, Hispanic patients.

PATIENTS: Four 2-year cohorts of patients aged 18-75 with DM and SBP $\geq 140 \mathrm{mmHg}$ on HTN drugs in year 1 and SBP measured 1 year later in year 2 .

INTERVENTION: Implementation of CCM for DM in January 2014 involved: electronic medical record revision, a DM registry, hypertension (HTN) treatment protocol, team education, performance feedback, and case management. MAIN MEASURE: SBP < $140 \mathrm{mmHg}$ in year 2 .

KEY RESULTS: Of 2354 patients, the mean age was 56.2 (SD 9.5), baseline SBP 153.8 (SD 14.9) $\mathrm{mmHg}$, and 79.8\% Hispanic. Last SBP $<140 \mathrm{mmHg}$ was $58.4 \%$ for cohort 1 (2012-2013) and 68.5\% for cohort 4 (2015-2016). Adjusted odds ratios (AORs) for SBP control versus cohort 1 were 1.35 (95\% CI 1.07, 1.69) for cohort 3 (2014-2015) and 2.13 (95\% CI 1.60, 2.80) for cohort 4. AORs for SBP control were reduced by $15 \%$ per HTN drug at baseline $(P=$ $0.001), 9 \%$ per HTN drug added at last SBP $(P=0.024)$, and $22 \%$ for multi-dose HTN drugs $(P=0.004)$. Among patients with persistent elevated SBP and represented in multiple cohorts, AORs for control were still over 2-fold higher for cohort 4 versus cohort 1.

CONCLUSIONS: After adopting the CCM for primarily Hispanic patients with DM, SBP control increased significantly despite treatment with fewer HTN drugs. Yet improvement took 3-4 years, suggesting that financial rewards for using the CCM to achieve improved clinical outcomes for low-income, minority patients may be delayed.

Electronic supplementary material The online version of this article (https://doi.org/10.1007/s11606-018-4526-3) contains supplementary material, which is available to authorized users.

Received March 27, 2018

Revised May 29, 2018

Accepted May 29, 2018

Published online June 8, 2018
KEY WORDS: chronic disease management; diabetes mellitus; patientcentered care; quality improvement.

J Gen Intern Med 33(9):1498-503

DOI: $10.1007 / \mathrm{s} 11606-018-4526-3$

(C) Society of General Internal Medicine 2018

\section{INTRODUCTION}

Myocardial infarction and all-cause mortality in persons with diabetes mellitus and systolic blood pressure (SBP) $\geq 140 \mathrm{mmHg}$ are significantly reduced by antihypertensive therapy. ${ }^{1}$ Yet racial-ethnic minorities are less likely to achieve hypertension control $^{2,3}$ even when being treated. ${ }^{4}$ To reduce disparities in quality of care and outcomes of patients with diabetes, experts have endorsed implementing the Chronic Care Model (CCM). ${ }^{5}$ Another systematic review of outcomes after implementing the CCM reported that processes of care improved within 1-2 years but intermediate clinical outcomes such as hemoglobin Alc did not significantly improve until 2-4 years afterward. ${ }^{6}$ Waiting years after investing in the CCM to receive incentives for meeting metrics for clinical outcomes may be especially burdensome for primary care practices serving vulnerable populations.

In a 4-year 1115 Medicaid waiver project in two primary care residency training practices serving low-income, predominantly Hispanic patients, we examined secular trends in achieving SBP control after implementing the CCM for diabetes. Evidence-based components of the $\mathrm{CCM}^{7}$ included the following: electronic medical record (EMR) redesign, creation of a diabetes registry, an antihypertensive management protocol, clinician and staff education, performance feedback, and case management support. In four consecutive two-cohorts, we studied patients with baseline $\mathrm{SBP} \geq 140 \mathrm{mmHg}$ while on treatment in year 1 and achievement of SBP control $(<$ $140 \mathrm{mmHg}$ ) per expert guidelines ${ }^{8,9}$ at the last SBP measurement in year 2. We also examined covariates associated with achievement of SBP control including patient demographics, clinical characteristics, and medication management. This analysis offers insights into the time to realize benefits from the CCM and factors that may influence achievement of hypertension control in a low-income, minority population. 


\section{METHODS}

\section{Setting and Patients}

The project was conducted for the Texas Healthcare Transformation and Quality Improvement Program (1115 Medicaid Waiver) in family medicine and general internal medicine residency training clinics in south central Texas. The 1115 Medicaid Waiver allowed the state of Texas to expand Medicaid managed care and services for the uninsured. The study practices served a largely uninsured, Hispanic patient population. Clinic faculty is stable but new residents arrive each year. From the electronic medical record (EMR) plus scheduling data, a new diabetes registry was created with patients meeting the following criteria: age 18 to 75 ; diabetes mellitus (one inpatient or two outpatient encounters with ICD-9-CM 250.xx or ICD-10 E10.xx or E11.xx) or a hemoglobin A1c $\geq 6.5 \%(\geq$ $48 \mathrm{mmol} / \mathrm{mol}$ ); and least one clinic visit from January 1, 2012 through December 31, 2016. The registry offered the following: demographics, ICD-9-CM and ICD-10 diagnoses from all encounters, prescribed medications, laboratory test results, preventive health care, insurance type, and health care utilization. ${ }^{10}$ Results of tests from outside laboratories were extracted from the companies' databases and added to the registry.

For 1115 Medicaid waiver diabetes quality metrics, the blood pressure goal was $<140 / 90$. Four cohorts of patients treated in consecutive year-pairs (e.g., 2012-2013; 20132014; 2014-2015, 2015-2016) were created with a SBP $\geq$ $140 \mathrm{mmHg}$ in year 1 while prescribed antihypertensive therapy and a SBP measured in clinic at least 12 months afterward. Because CCM program was implemented in 2014, the first and second cohorts served as a baseline before full implementation.

\section{Chronic Care Model Implementation}

The CCM had six major components. First, a diabetes registry (described above) was developed and continuously updated to generate daily reports for scheduled patients with most recent blood pressure, hemoglobin A1c, LDL cholesterol, immunizations, patient adherence to scheduled visits, urgent care encounters, and hospitalizations. Second, clinic directors, researcher-clinicians, and pharmacists adapted an evidencebased hypertension treatment protocol ${ }^{11}$ for patients with diabetes, prioritizing accessible drugs on local formularies while minimizing medication burden ${ }^{12-14}$ and costs ${ }^{15}$ (Supplemental Figure 1-online). Third, a retrievable field for repeat blood pressure measurements was added to the EMR when the first measurement was elevated per HTN guidelines. ${ }^{8,9}$ Fourth, clinicians and staff were trained in using the protocol, accurately measuring blood pressure, and supporting medication adherence through reminders and patient education. Fifth, clinicians received a biannual summary of their patients' metrics on blood pressure, hemoglobin A1c, and LDL cholesterol with abnormal values highlighted. Sixth, trained bilingual case managers assisted patients with uncontrolled hypertension in clinic or by telephone. They addressed the following: obtaining medications and timely refills, visit adherence, transportation assistance, obtaining blood tests, and lifestyle changes to improve diabetes and hypertension control.

\section{Analyses}

Dependent Variable. For each 2-year cohort, the dichotomized outcome was controlled SBP defined as $<140 \mathrm{mmHg}$ at last clinic measurement in year 2 .

Independent Variables. For each of the four cohorts, demographic, clinical, and health care variables were extracted from the diabetes registry. Demographics included age, race/ethnicity, and sex. Clinical variables included baseline SBP in year 1 categorized in $10 \mathrm{mmHg}$ increments from 140 to $\geq 170 \mathrm{mmHg}$; mean hemoglobin A1c in year 1 categorized as $<7,7-7.9,8-8.9, \geq 9 \%(<53,53-63,64-74, \geq$ $75 \mathrm{mmol} / \mathrm{mol}$ ) or missing, and Elixhauser comorbidity index with up to 19 comorbidities. ${ }^{10}$ Health care variables for each 2-year cohort included the following: observation time (days from first elevated SBP in year 1 until last SBP in year 2), insurance type, scheduled primary care and specialty clinic visits, and adherence to scheduled visits $[<60 \%, 60-<75 \%, \geq$ $75 \%$ ]. ${ }^{10}$ Prescribed antihypertensive drugs were grouped by class (i.e., angiotensin converting enzyme inhibitors, angiotensin II receptor blockers, anti-adrenergic agents, betablockers, calcium channel blockers, diuretics, and vasodilators) and totaled at first elevated SBP in year 1. To evaluate secular changes in treatment, the number of prescribed antihypertensive classes was measured at the last SBP in year 3 and the difference from year 1 determined. A binary variable was created for any prescribed antihypertensive drug with multiple daily doses. From a REDCap database of case management services, a four-level categorical variable was developed for services provided in year 1 , year 2 , both years, or none.

Statistical Analyses. Descriptive characteristics for the four cohorts were compared using the chi-square test or Fisher's exact test for categorical variables and Kruskal-Wallis $H$ test for continuous variables. In mixed effects logistic regression accounting for correlations introduced by patients in multiple cohorts, predictors for controlled last SBP in each cohort were examined. The full model included all independent variables described above and indicator for each cohort. We also examined first-order interactions between each independent variable with time (i.e., the cohort variable) to examine whether the effect was modified by time. Clinical judgment guided removal of non-significant main effects and interaction terms. A post hoc analysis was conducted adjusting for patients represented in only one cohort versus those in multiple cohorts whose blood pressure likely stayed uncontrolled. Bivariate associations for single versus multi-cohort patients were examined with the outcome. The same final mixed effects model predicting SBP control was fitted, adding a variable for 
patients in a single or multiple cohorts and an interaction term with the four-level cohort variable.

All analyses were performed using Stata/SE (version 14.1, 2015, StataCorp LP, College Station, TX). This study was approved by the Institutional Review Boards of both the University of Texas Health Science Center at San Antonio and University Health System (HSC20140003N).

\section{RESULTS}

Among all 5990 patients with diabetes in the study clinics over a 5-year timeframe (January 1, 2012 to December 31, 2016), $3691(61.6 \%)$ had blood pressure measurements in consecutive years and, of these, 2354 (63.8\%) had uncontrolled SBP $(\geq 140 \mathrm{mmHg})$ in year 1 while on treatment. These patients were characterized by mean age 56.2 (SD 9.5), baseline SBP $153.8 \mathrm{mmHg}$ (SD 14.9), and 79.8\% Hispanic. Because the CCM was initiated in January 2014, cohort 1 (2012-13) and year 1 for cohort 2 (2013-2014) reflect performance before implementation. Each four 2-year cohorts had at least 1000 patients (Table 1). Over time, the proportion of uninsured persons declined after the Affordable Care Act but proportions on Medicaid and Medicare remained relatively stable (Table 1). At baseline, the SBP declined slightly from 154.2 to $152.6 \mathrm{mmHg}$ for successive 2-year cohorts and the proportion with a SBP from 140 to $149 \mathrm{mmHg}$ increased from 51.3 to $57.8 \%$. On the other hand, mean baseline hemoglobin A1c increased from 7.9 to $8.3 \%$ (63 to $67 \mathrm{mmol} / \mathrm{mol}$ ) for successive cohorts. The Elixhauser comorbidity score and mean follow-up time were similar for all cohorts. Successive cohorts had fewer visits in year 1 but adherence to scheduled visits did not change significantly. Notably, approximately one-fifth of patients had low visit adherence $(<60 \%$ of visits attended).

The mean antihypertensive drug classes prescribed in year 1 declined slightly from cohort 1 to 4 (Table 1). At the time of the last SBP in year 2, cohorts 1 and 2 were prescribed more antihypertensive medications but cohorts 3 and 4 were prescribed fewer. Multiple daily dose drugs declined significantly over time from 45 to $35 \%$. Case management was initiated during cohort 2 and fully operative in cohorts 3 and 4 when it was provided to over half of the patients.

SBP control $(\leq 140 \mathrm{mmHg})$ at the last measurement in year 2 increased significantly $(P<0.001)$ from 58.4 to $68.5 \%$ from cohort 1 to 4 (Fig. 1). Mean last SBP also declined $(P=0.01)$ for cohorts 1 to 4 (137.0 (SD 18.5), 137.7 (SD 19.8), 136.4 (SD 19.1), 135.4 (SD 19.4), respectively). In mixed effects logistic regression with cohort 1 as the reference, the adjusted odds ratios (AORs) for SBP control in year 2 were $1.35(95 \%$ CI $1.07-1.69 ; P=0.01)$ for cohort 3 and 2.13 (95\% CI 1.60 $2.80 ; P<0.001)$ for cohort 4 (Table 2). Older age and Black race (versus Hispanic) were negatively associated with control (both $P<0.01$ ) while Medicare insurance was positively associated versus uninsured $(P=0.04)$. Mean hemoglobin A1c $\geq 9 \%$ ( $\geq 75 \mathrm{mmol} / \mathrm{mol}$ ) in year 1 was associated with a lower
AOR $(0.79,95 \%$ CI $0.65,0.96)$ of SBP control in year 2 versus $\mathrm{A} 1 \mathrm{c}<7 \%(<53 \mathrm{mmol} / \mathrm{mol})(P=0.02)$. Increasing baseline levels of SBP were associated with monotonically lower AORs of achieving control in year 2 (all $P<0.0001$ ). When at least $75 \%$ of scheduled visits were kept, the AOR of SBP control in year 2 was higher 1.33 (95\% CI 1.08, 1.64) compared with $<60 \%(P=0.007)$.

AORs of SBP control were reduced by $15 \%$ for each additional antihypertensive medication class prescribed at baseline ( $P=0.001)$ and $9 \%$ lower for each medication added by the last $\operatorname{SBP}(P=0.024)$. Multiple daily dose drugs were associated with a $22 \%$ reduction in the AOR of SBP control $(P=0.004)$. Case management services were associated with significantly lower AORs of SBP control except when only delivered in the first of the year-pairs.

In a post hoc analysis (Table 3 ), we examined SBP control among patients in only one cohort $(N=1101)$ and those in multiple cohorts $(N=1253)$. For the single cohort group, proportions with SBP control in year 2 for cohorts 1-4 were $63.8,73.7,74.2$, and $70.1 \%$, respectively. For the multi-cohort group, these values for cohorts $1-4$ were 55.9, 54.2, 59.2, and $67.9 \%$, respectively. A significant $(P=0.012)$ interaction appeared between single versus multi-cohort patients and the cohort variable in the fully adjusted model. Among single cohort patients, the AORs of controlled SBP in year 2 were increased for cohorts 2 to 4 versus cohort 1 (all $P<0.05$ ) (Table 3). Among the multiple cohort group, SBP control was significantly increased only for cohorts 3 and 4 (both $<$ $0.02)$.

\section{CONCLUSIONS}

After implementation the Chronic Care Model (CCM) for diabetes in two primary care clinics serving an indigent, Hispanic majority population, systolic blood pressure (SBP) control $(<140 \mathrm{mmHg})$ improved markedly among patients whose baseline SBP was $\geq 140 \mathrm{mmHg}$ while on antihypertensive therapy. Among successive 2-year patient cohorts from 2012 through 2016, controlled SBP in year 2 rose from $58 \%$ of cohort $1(N=1151$ to $68 \%$ of cohort $4(N=1080)$. By comparison, 53\% of Hispanics in a 2008 national diabetes study had controlled hypertension ${ }^{16}$ and, in a national study from 2015 to 2016, only $45 \%$ of Hispanics with diagnosed hypertension were controlled $(<140 / 90 \mathrm{mmHg}) .{ }^{17}$ We studied SBP control as our outcome because it is the dominant abnormality in hypertension. We also focused on a challenging group of patients whose blood pressure was uncontrolled at baseline despite treatment, making the $68 \%$ control achieved in year 2 for cohort 4 even more impressive.

Chin and colleagues' review of outcomes from implementing the CCM in practices serving indigent populations reported that glycemic control for diabetes did not significantly improve until 2-4 years after implementation. ${ }^{6} \mathrm{Sim}-$ ilarly, we found that SBP control did not improve until 3- 
Table 1 Characteristics of four cohorts of patients with diabetes and uncontrolled systolic blood pressure $(\geq 140 \mathrm{mmHg})$ at baseline

\begin{tabular}{|c|c|c|c|c|c|}
\hline \multirow[t]{2}{*}{ Study variables } & \multirow{2}{*}{$\begin{array}{l}\begin{array}{l}\text { Cohort } 1(2012- \\
\text { 2013) }\end{array} \\
N=1151\end{array}$} & \multirow{2}{*}{$\begin{array}{l}\text { Cohort } 2(2013- \\
\text { 2014) } \\
N=1199\end{array}$} & \multirow{2}{*}{$\begin{array}{l}\text { Cohort } 3 \text { (2014- } \\
\text { 2015) } \\
N=1106\end{array}$} & \multirow{2}{*}{$\begin{array}{l}\text { Cohort } 4 \text { (2015- } \\
\text { 2016) } \\
N=1080\end{array}$} & \multirow[t]{2}{*}{$P$ value } \\
\hline & & & & & \\
\hline \multicolumn{6}{|l|}{ Demographics } \\
\hline Age, mean $(\mathrm{SD})$ years & $57.3(9.5)$ & $57.3(9.3)$ & $58.1(9.1)$ & $57.9(9.2)$ & $0.07 \dagger$ \\
\hline Women, $n(\%)$ & $710(61.7)$ & $715(59.6)$ & $645(58.3)$ & $653(60.5)$ & $0.42 \ddagger$ \\
\hline Race-ethnicity, $n(\%)$ & & & & & $0.63 t$ \\
\hline Hispanic & 908 (78.9) & $938(78.2)$ & $876(79.2)$ & $871(80.7)$ & \\
\hline Non-Hispanic White & $117(10.2)$ & $128(10.7)$ & $110(10.0)$ & $87(8.1)$ & \\
\hline Black & $97(8.4)$ & $106(8.8)$ & $90(8.1)$ & $100(9.3)$ & \\
\hline Other & $29(2.5)$ & $27(2.3)$ & $30(2.7)$ & $22(2.0)$ & \\
\hline Insurance, $n(\%)$ & & & & & $<0.001 \dagger$ \\
\hline Uninsured & $532(46.2)$ & $580(48.4)$ & $429(38.8)$ & $414(38.3)$ & \\
\hline Medicaid & $161(14)$ & $159(13.3)$ & $147(13.3)$ & $158(14.6)$ & \\
\hline Medicare & $424(36.8)$ & $415(34.6)$ & $432(39.1)$ & $402(37.2)$ & \\
\hline Private/health maintenance organization & $34(3)$ & $45(3.8)$ & $98(8.9)$ & $106(9.8)$ & \\
\hline \multicolumn{6}{|l|}{ Clinical } \\
\hline Baseline SBP* (mmHg), mean (SD) & $154.2(14.5)$ & $153.9(14.3)$ & $152.4(13.7)$ & $152.6(14.4)$ & $<0.001 \dagger$ \\
\hline \multicolumn{6}{|l|}{ Baseline SBP* categories (mmHg), $N(\%)$} \\
\hline 140 to $<150$ & $590(51.3)$ & $606(50.5)$ & $600(54.3)$ & $624(57.8)$ & \\
\hline 150 to $<160$ & $262(22.8)$ & $306(25.5)$ & $302(27.3)$ & $216(20)$ & \\
\hline 160 to $<170$ & $138(12)$ & $129(10.8)$ & $87(7.9)$ & $100(9.3)$ & \\
\hline$\geq 170$ & $161(14)$ & $158(13.2)$ & $117(10.6)$ & $140(13)$ & \\
\hline Hemoglobin A1c in baseline year, mean (SD) & $\begin{array}{l}7.9(1.9) 63 \\
\mathrm{mmol} / \mathrm{mol}\end{array}$ & $\begin{array}{l}7.8(1.9) 62 \\
\mathrm{mmol} / \mathrm{mol}\end{array}$ & $\begin{array}{l}8.0(2.1) 64 \\
\mathrm{mmol} / \mathrm{mol}\end{array}$ & $\begin{array}{l}8.3(2.2) 67 \\
\mathrm{mmol} / \mathrm{mol}\end{array}$ & $<0.001 \ddagger$ \\
\hline \multirow{2}{*}{\multicolumn{6}{|c|}{$\begin{array}{l}\text { Ellxhauser comorbiarty score, mean (SD) } \\
\text { Health care utilization }\end{array}$}} \\
\hline & & & & & \\
\hline Days from first high $\mathrm{SBP}^{*}$ to last $\mathrm{SBP}$, mean $(\mathrm{SD})$ & $558(98)$ & $556(97)$ & $547(101)$ & $545(91)$ & $0.002 \dagger$ \\
\hline \# visits attended in year 1 , mean (SD) & $12.6(6.4)$ & $10.6(5.4)$ & $10.6(5.4)$ & $11.4(5.4)$ & $<0.001 t$ \\
\hline Adherence to scheduled visits in year 1 & & & & & $0.50 \dagger$ \\
\hline Low $(<60 \%)$ & $225(19.6)$ & $240(20.0)$ & $198(17.9)$ & $204(18.9)$ & \\
\hline Moderate $(60$ to $<75 \%)$ & $424(36.8)$ & $408(34.0)$ & $401(36.3)$ & $356(33.0)$ & \\
\hline High $(\geq 75 \%)$ & $502(43.6)$ & $551(46.0)$ & $507(45.8)$ & $520(48.2)$ & \\
\hline \multicolumn{6}{|l|}{ Hypertension management } \\
\hline $\begin{array}{l}\text { Antihypertensive drug classes prescribed at first } \\
\text { elevated SBP* mean (SD) }\end{array}$ & $1.7(1.2)$ & $1.6(1.1)$ & $1.6(1.1)$ & $1.6(1.1)$ & $0.07 \dagger$ \\
\hline $\begin{array}{l}\text { Change in antihypertensive drug classes prescribed } \\
\text { at last SBP*, mean (SD) }\end{array}$ & $0.15(1.0)$ & $0.1(1.0)$ & $-0.07(1.0)$ & $-0.23(1.1)$ & $<0.001 \ddagger$ \\
\hline $\begin{array}{l}\text { Any antihypertensive drug with multiple daily } \\
\text { doses } N(\%)\end{array}$ & $514(44.7)$ & $498(41.5)$ & $430(38.9)$ & $382(35.4)$ & $<0.001 \ddagger$ \\
\hline Case management services, $N(\%)$ & & & & & $<0.0001$ \\
\hline None & $1151(100.0)$ & $857(71.5)$ & $576(52.1)$ & $474(43.9)$ & \\
\hline Only in year 2 & 0 & $342(28.5)$ & 207 (18.7) & $225(20.8)$ & \\
\hline Only in year 1 & 0 & $0(0.00)$ & $171(15.5)$ & 145 (13.4) & \\
\hline In years 1 and 2 & 0 & $0(0.00)$ & $152(13.7)$ & $236(21.9)$ & \\
\hline
\end{tabular}

*SBP systolic blood pressure

†ruskal-Wallis H test

$\neq$ Chi-squared test

4 years after CCM implementation, with over 2-fold higher AORs of SBP control for cohort 4 versus cohort 1.

Implementation of the $\mathrm{CCM}$ requires both time and money to create a disease registry, modify the EMR to support quality of care, develop and train clinicians and staff, provide quality of care feedback, and offer case management. For example, our program used 2.0 FTE community health workers (CHWs) at $\$ 33,000 /$ each ( $\$ 66,000$ total), a 1.0 FTE RN $(\$ 60,000)$ as well as 0.2 FTE senior health services researcher $(\$ 57,000), 1.0$ FTE project coordinator $(\$ 45,000)$, and 0.6 FTE data analyst $(\$ 37,000)$. Although ultimately, the CCM will be supported only by clinical personnel and a data analyst, this is still costly. In a cost-effectiveness analysis, CCM in a military diabetes clinic was estimated to cost $\$ 45,495$ per quality-adjusted life-year (QALY) from a health system perspective. ${ }^{18}$ But this practice redesign can be promoted with

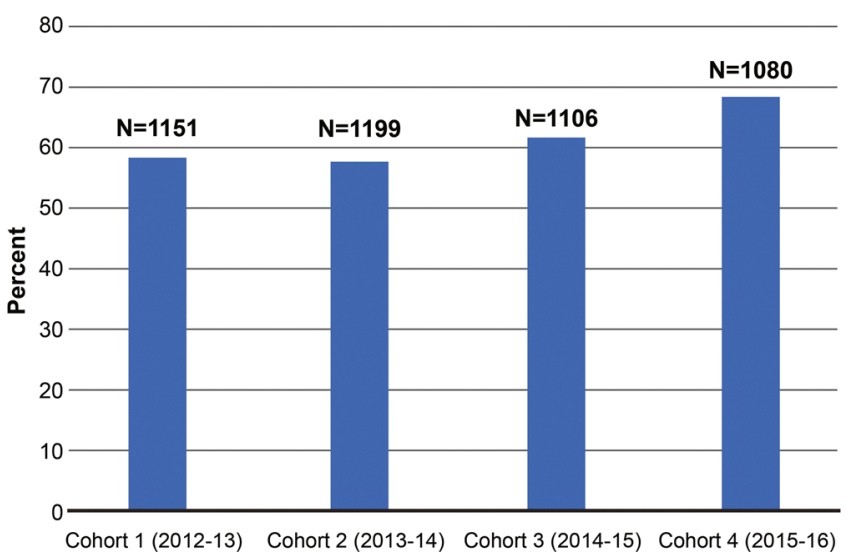

Fig. 1 Proportion of patients in each 2-year cohort with systolic blood pressure control $(<140 \mathrm{mmHg})$ on the last measurement in year 2. 
Table 2 Adjusted associations of patient characteristics with controlled systolic blood pressure $(<140 \mathrm{mmHg})$ at last measurement in year 2

\begin{tabular}{|c|c|c|}
\hline Study variables & $\begin{array}{l}\text { Adjusted odds ratio } \\
(95 \% \mathrm{CI}) \dagger\end{array}$ & $P$ value \\
\hline \multicolumn{3}{|l|}{ Patient cohort } \\
\hline $2012-2013$ & 1 & \\
\hline 2013-2014 & $1.11(0.91,1.35)$ & 0.31 \\
\hline $2014-2015$ & $1.35(1.07,1.69)$ & 0.01 \\
\hline 2015-2016 & $2.13(1.60,2.83)$ & $\begin{array}{l}< \\
0.0001\end{array}$ \\
\hline \multicolumn{3}{|l|}{ Age } \\
\hline Per 1-year increase & $0.98(0.97,0.99)$ & 0.001 \\
\hline \multicolumn{3}{|l|}{ Sex } \\
\hline Male & 1 & \\
\hline Female & $0.9(0.76,1.05)$ & 0.18 \\
\hline \multicolumn{3}{|l|}{ Race-ethnicity } \\
\hline Hispanic & 1 & \\
\hline Non-Hispanic White & $0.93(0.71,1.21)$ & 0.58 \\
\hline Black & $0.67(0.50,0.89)$ & 0.005 \\
\hline Other & $0.68(0.41,1.13)$ & 0.13 \\
\hline \multicolumn{3}{|l|}{ Insurance } \\
\hline Uninsured & 1 & \\
\hline Medicaid & $1.1(0.86,1.4)$ & 0.43 \\
\hline Medicare & $1.23(1.01,1.5)$ & 0.037 \\
\hline $\begin{array}{l}\text { Private/health maintenance } \\
\text { organization }\end{array}$ & $0.91(0.65,1.27)$ & 0.57 \\
\hline \multicolumn{3}{|l|}{ Elixhauser comorbidity score } \\
\hline Per 1-point increase & $1.0(0.97,1.03)$ & 0.92 \\
\hline \multirow{2}{*}{\multicolumn{3}{|c|}{$\begin{array}{l}\text { Baseline mean hemoglobin a1c (\%) } \\
<7(<53 \mathrm{mmol} / \mathrm{mol})\end{array}$}} \\
\hline & & \\
\hline Missing & $0.97(0.66,1.42)$ & 0.86 \\
\hline $7-7.9(53-63 \mathrm{mmol} / \mathrm{mol})$ & $0.86(0.70,1.06)$ & 0.17 \\
\hline 8-8.9 (64-8.9 mmol/mol) & $0.94(0.74,1.19)$ & 0.62 \\
\hline$\geq 9(\geq 75 \mathrm{mmol} / \mathrm{mol})$ & $0.79(0.65,0.96)$ & 0.02 \\
\hline \multicolumn{3}{|l|}{ Baseline SBP* (mmHg) } \\
\hline 140 to $<150$ & 1 & \\
\hline 150 to $<160$ & $0.73(0.61,0.87)$ & $\begin{array}{l}< \\
0.0001\end{array}$ \\
\hline 160 to $<170$ & $0.59(0.46,0.75)$ & $\begin{array}{l}< \\
0.0001\end{array}$ \\
\hline$\geq 170$ & $0.4(0.32,0.5)$ & $\begin{array}{l}<.0001 \\
0.0001\end{array}$ \\
\hline \multicolumn{3}{|l|}{ Adherence to scheduled visits } \\
\hline Low $(<60 \%)$ & 1 & \\
\hline Moderate $(60$ to $<75 \%)$ & $1.19(0.96,1.46)$ & 0.11 \\
\hline High $(\geq 75 \%)$ & $1.33(1.08,1.64)$ & 0.007 \\
\hline \multicolumn{3}{|c|}{ Antihypertensive drug classes prescribed at first elevated $\mathrm{SBP}^{*}$} \\
\hline Per each additional class & $0.85(0.78,0.93)$ & 0.001 \\
\hline $\begin{array}{l}\text { Change in antihypertensive drug } \\
\text { classes at last SBP* }\end{array}$ & & \\
\hline Per each additional category & $0.91(0.84,0.99)$ & 0.024 \\
\hline \multicolumn{3}{|c|}{ Prescribed antihypertensive drug with multiple daily doses } \\
\hline Yes & $0.78(0.65,0.92)$ & 0.004 \\
\hline No & 1 & \\
\hline \multicolumn{3}{|l|}{ Case management services } \\
\hline None & 1 & \\
\hline Only in year 2 & $0.58(0.47,0.71)$ & $\begin{array}{l}< \\
0.0001\end{array}$ \\
\hline Only in year 1 & $1.21(0.87,1.68)$ & 0.25 \\
\hline In years 1 and 2 & $0.50(0.37,0.68)$ & $\begin{array}{l}< \\
0.0001\end{array}$ \\
\hline
\end{tabular}

*SBP systolic blood pressure

† Mixed effects logistic regression

financial incentives. In a trial of pay-for-performance incentives for small New York City practices, significant improvement in blood pressure control in patients with diabetes appeared in less than 1 year but only $26 \%$ achieved control. ${ }^{19}$ More financially sound practices serving insured patients can better afford the cost of the CCM. Over $85 \%$ of all patients served after practice improvements in Kaiser Permanente
Table 3 Adjusted odds ratio* for controlled last systolic blood pressure for model with the interaction term for cohort year and patients in one cohort versus in multiple cohorts

\begin{tabular}{lll}
\hline \hline Interaction & Adjusted odds ratio (95\% CI) & $P$ value \\
\hline Single cohort & & \\
2012-2013 & Reference & \\
$2013-2014$ & $1.77(1.16-2.71)$ & 0.008 \\
$2014-2015$ & $1.64(1.02-2.64)$ & 0.041 \\
2015-2016 & $1.69(1.10-2.60)$ & 0.016 \\
Multiple cohorts & Reference & \\
2012-2013 & $1.05(0.85-1.31)$ & 0.64 \\
2013-2014 & $1.36(1.06-1.74)$ & 0.015 \\
$2014-2015$ & $2.32(1.70-3.15)$ & $<0.0001$ \\
2015-2016 &
\end{tabular}

*Mixed effects logistic regression including all variables in Table 2, a binary variable for single versus multiple cohort patients and the interaction with the four-level cohort year variable

Southern California had controlled hypertension. ${ }^{20}$ In our primarily Medicaid and uninsured, minority population, CCM implementation was made possible by the 1115 Medicaid waiver to Texas to defray the costs.

A key feature of our CCM was implementing a protocol for hypertension treatment in patients with diabetes following the example of an evidence-based protocol in an insured population. ${ }^{11}$ By adapting this protocol to feature effective, lower cost medications, improved control was achieved despite prescribing fewer drugs. We also avoided drugs requiring multiple daily doses (e.g., enalapril versus lisinopril) which were associated with a $22 \%$ lower AORs of SBP control. These results lend support to an international initiative to improve blood pressure control by implementing an evidence-based treatment protocol. ${ }^{21}$

Even with CCM, SBP control was less likely for older patients, Blacks versus Hispanics, and patients with mean baseline hemoglobin A1c $\geq 9 \%(\geq 75 \mathrm{mmol} / \mathrm{mol})$ versus $<$ $7 \%(<53 \mathrm{mmol} / \mathrm{mol})$. Further, we found that patients who kept at least $75 \%$ of scheduled visits were more likely to be controlled than those keeping $<60 \%$. Similarly, poor adherence to visits $(<70 \%)$ in a large HMO was associated with significantly poorer glycemic control. ${ }^{22}$ Although case management was not associated with improved control, this finding may represent confounding by indication because case managers target patients who continue to have uncontrolled hypertension or diabetes. We also found that patients represented in only one cohort showed improvement more rapidly, with SPB control increasing from 63 to $73 \%$ by cohort 2 (the first year of the CCM). This likely represents an artifact of study eligibility criteria because patients who remain uncontrolled continue to appear in multiple cohorts. But even this more challenging group showed marked improvement in SBP control in the later years of the study.

In regard to limitations, these primary care practices serving Hispanic-majority patients may not be generalizable to other settings serving minority groups. Second, this 5-year study may reflect secular changes in hypertension control but 
national studies do not show similar improvement in hypertension control over this timeframe. The National Center for Health Statistics reported that $53.3 \%$ of adults were controlled $(<140 / 90 \mathrm{mmHg}$ ) in $2010-2011,54.0 \%$ in $2013-2014$, and $50.8 \%$ in $2015-2016 .^{3,17}$ Third, blood pressures were obtained from office readings that were repeated when elevated per guidelines but did not reflect home monitoring. ${ }^{9}$ Fourth, we cannot distinguish CCM features that were more effective. Lastly, only two-thirds of patients achieved SBP control but we focused only on persons with uncontrolled SBP while on treatment.

Developing effective models of hypertension care for Hispanics with diabetes is a national priority because of the rising prevalence of diabetes complicated by hypertension in this US population, from $4.8 \%$ in $1999-2000$ to $7.7 \%$ in $2011-2012,{ }^{21}$ and increasing hypertension-related death. ${ }^{22}$ This study offers promising evidence supporting the CCM to improve SBP control in our minority patient population. However, our practice redesign was supported by external funding so it is important for administrators and policymakers to understand that it may take 3 to 4 years to observe clinical benefits from this investment in practices serving low-income, minority populations.

Corresponding Author: Barbara J. Turner, MD, MSED; Center for Research to Advance Community Health (ReACH)UT Health San Antonio, San Antonio, TX, USA (e-mail: turner@uthscsa.edu).

Contributors The authors wish to thank Sarah Lill for her administrative support for this project.

Funding Research reported in this publication was supported by the Center for Medicare and Medicaid Services 1115 Medicaid waiver \#085144601.1.2

\section{Compliance with Ethical Standards}

This study was approved by the Institutional Review Boards of both the University of Texas Health Science Center at San Antonio and University Health System (HSC20140003N).

Conflict of Interest: The authors declare that they do not have a conflict of interest.

Prior Presentation: Parts of the results of this study were presented in poster form at the Society of General Internal Medicine Annual Meeting; April 19 to April 22, 2017

\section{REFERENCES}

1. Brunström M, Carlberg B. Effect of antihypertensive treatment at different blood pressure levels in patients with diabetes mellitus: systematic review and meta-analyses. BMJ. 2016;352:1717.

2. Beckles GL, Chou C. Disparities in the prevalence of diagnosed diabetes-United States, 1999-2002 and 2011-2014. MMWR. Morb Mort Wkly Rep. 2016;65(45):1265-1269.

3. Yoon SS, Carroll MD, Fryar CD. Hypertension prevalence and control among adults: United States, 2011-2014. NCHS Data Brief. 2015;220:18.
4. Redmond N, Baer HJ, Hicks LS. Health behaviors and racial disparity in blood pressure control in the national health and nutrition examination survey. Hypertension. 2011;57(3):383-389.

5. American Diabetes Association. Promoting health and reducing disparities in populations. Diabetes Care. 2017;40(1):S6-S10.

6. Chin MH. Quality improvement implementation and disparities: the case of the health disparities collaboratives. Med Care. 2011;49:S65-S71.

7. Coleman K, Austin BT, Brach C, Wagner EH. Evidence on the Chronic Care Model in the new millennium. Health Aff (Millwood). 2009;28(1):7585.

8. James PA, Oparil S, Carter BL, et al. 2014 evidence-based guideline for the management of high blood pressure in adults: Report from the panel members appointed to the Eighth Joint National Committee (JNC 8). JAMA. 2014;311(5):507-520.

9. de Boer IH, Bangalore S, Benetos A, Davis AM, Michos ED, Muntner P, Rossing P, Zoungas S, Bakris G. Diabetes and hypertension: a position statement by the American Diabetes Association. Diabetes Care. 2017;40(9):1273-1284.

10. Zullig LL, Liang $\mathbf{Y}$, Vale Arismendez S, Trevino A, Bosworth HB, Turner BJ. Trajectory of systolic blood pressure in a low-income, racialethnic minority cohort with diabetes and baseline uncontrolled hypertension. J Clin Hypertens (Greenwich). 2017;19(7):722-730.

11. Jaffe MG, Lee GA, Young JD, Sidney S, Go AS. Improved blood pressure control associated with a large-scale hypertension program. JAMA. 2013;310(7):699-705.

12. Gerbino PP, Shoheiber $\mathbf{O}$. Adherence patterns among patients treated with fixed-dose combination versus separate antihypertensive agents. Am J Health Syst Pharm. 2007;64(12):1279-1283.

13. Hagendorff A, Freytag S, Müller A, Klebs S. Pill burden in hypertensive patients treated with single-pill combination therapy-an observational study. Adv Ther. 2013;30(4):406-419.

14. Tiktin M, Celik S, Berard $\mathbf{L}$. Understanding adherence to medications in type 2 diabetes care and clinical trials to overcome barriers: a narrative review. Curr Med Res Opin. 2016;32(2):277-87.

15. Taitel M, Fensterheim L, Kirkham H, Sekula R, Duncan I. Medication days' supply, adherence, wastage, and cost among chronic patients in Medicaid. Medicare Medicaid Res Rev. 2012;2(3):E1-E13.

16. Belue R, Oluwole AN, Degboe ANF, Figaro MK. Hypertension control in ambulatory care patients with diabetes. Am J Manag Care. 2012;18(1):17-23.

17. Fryar CD, Ostchega Y, Hales CM, Zhang G. Hypertension prevalence and control among adults: United States, 2015-2016. NCHC Data Brief. 2017;(289): 1-8.

18. Kuo S, Bryce CL, Zgibor JC, Wolf DL, Roberts MS, Smith KJ. Costeffectiveness of implementing the chronic care model for diabetes care in a military population. J Diabetes Sci Technol. 2011;5(3):501-513.

19. Bardach NS, Wang JJ, De Leon SF, et al. Effect of pay-for-performance incentives on quality of care in small practices with electronic health records: a randomized trial. JAMA. 2013;310(10):1051-1059.

20. Sim JJ, Handler J, Jacobsen SJ, Kanter MH. Systemic implementation strategies to improve hypertension: The Kaiser Permanente Southern California experience. Can J Cardiol. 2014;30(5):544-52.

21. Patel P, Ordunez P, DiPette D, et al. Improved blood pressure control to reduce cardiovascular disease morbidity and mortality: the standardized hypertension treatment and prevention project. J Clin Hypertens (Greenwich). 2016;18(12):1284-1294.

22. Karter AJ, Parker MM, Moffet HH, et al. Missed appointments and poor glycemic control: an opportunity to identify high-risk diabetic patients. Med Care. 2004;42(2):110-115. 